\title{
Closing the Gap: Identifying Rates and Reasons for Nonadherence in a Specialty Population
}

\author{
Dominic Paolella, PharmD; Elizabeth Cherry, PharmD; Jacob A. Jolly, PharmD, CSP; \\ Joshua DeClercq, MS; Leena Choi, PhD; and Autumn Zuckerman, PharmD, BCPS, CSP
}

\begin{abstract}
BACKGROUND: Adherence to specialty and nonspecialty medications is often calculated using pharmacy claims data. However, specialty medication regimens are complex and may require periods of intentional gaps in therapy. Common adherence calculations are insufficient in identifying reasons for gaps in therapy. Because adherence reporting is a growing measure of quality care for specialty pharmacy accreditation and payer and manufacturer contracts, a better understanding of the rates and reasons for nonadherence within a specialty population is needed.
\end{abstract}

OBJECTIVE: To identify rates and reasons for misidentified and true nonadherence in patients who are prescribed specialty medications.

METHODS: A single center, retrospective cohort study was conducted using pharmacy claims data between March 2017 and February 2018. Medication adherence was calculated using proportion of days covered (PDC). Electronic medical records of a random $10 \%$ sample of nonadherent patients ( $P D C<80 \%$ ) were manually reviewed to identify reasons for nonadherence. Patients were then classified as either (a) misidentified as nonadherent (i.e., a provider-directed discontinuation or disruption of treatment that varies from the prescribed administration schedule or transfer of the prescription to an external pharmacy) or (b) truly nonadherent (discontinuation or disruption of treatment that varies from the prescribed administration instruction that is not directed or recommended by the provider or health care team).

RESULTS: Of the 7,488 included prescription records from 18 specialty areas, 1,059 met criteria for nonadherence. 105 prescription records (representing 105 unique patients) were manually reviewed; most of these patients $(58 \%)$ were truly nonadherent, driven by inability to contact patients for refills $(59 \%)$. However, $40 \%$ were misidentified as nonadherent, most due to provider-directed medication holding (69\%). Two percent of patients were nonadherent for unknown reasons.

CONCLUSIONS: Many patients classified as nonadherent based on pharmacy claims experienced gaps in therapy due to medically appropriate reasons. Methods to better measure and identify true nonadherence are needed to efficiently and adequately affect specialty medication adherence behavior.

J Manag Care Spec Pharm. 2019;25(11):1282-88

Copyright $\odot 2019$, Academy of Managed Care Pharmacy. All rights reserved.

\section{What is already known about this subject}

Nonadherence to prescribed medication is associated with poor clinical outcomes, progression of disease, and an economic burden in health care costs.

- A growing number of adherence metrics are being used in practice for contracting and accreditation, with financial implications for pharmacies that do not meet adherence thresholds.

- Several calculations exist to assess adherence using pharmacy claims, but current calculations are insufficient in recognizing medically appropriate reasons for gaps in therapy.

\section{What this study adds}

Analyzing 7,488 prescriptions, this study is the first to provide rates of adherence across several specialty clinics.

This study provides needed insight on rates and reasons of misidentified nonadherence and true nonadherence in specialty medication therapy, using data collected from medical and pharmacy records of 105 patients deemed nonadherent based on the commonly used proportion of days covered calculation.

Study findings demonstrate that relying on pharmacy claims calculations may inadequately portray true adherence behavior and is ineffective and unrealistic for certain specialty disease states.

A dherence to specialty medication is crucial for managing specialty diseases. The rate of medication nonadherence in a population of patients with a variety of specialty diseases has not been reported. However, more than half of patients prescribed nonspecialty medications for chronic conditions are nonadherent. ${ }^{1-3}$ The high costs of specialty medications, some exceeding $\$ 100,000$ per year, emphasize the importance of adherence to ameliorate medical costs in these conditions. ${ }^{4}$ Specialty pharmacy models aim to optimize use of specialty medications and ensure patient adherence, resulting in the anticipated clinical benefit of these expensive treatments. ${ }^{4}$ Previous research shows higher adherence rates in patients using a specialty pharmacy compared with a retail pharmacy. 5 Specialty pharmacies must be vigilant in efforts to improve adherence by identifying true gaps in therapy and designing appropriate interventions to address these gaps. In doing so, specialty pharmacies will be able to better manage pharmacy costs and patient safety and outcomes. 
As the field of specialty pharmacy continues to grow in the United States, published rates of adherence within specific specialty diseases are emerging, ranging from $44 \%-61 \%$ in pulmonary arterial hypertension and 54\%-94\% in multiple sclerosis to $89.3 \%$ in oncology.$^{6-9}$ Benchmarks for adherence in certain specialty diseases (rheumatoid arthritis, multiple sclerosis, and human immunodeficiency virus [HIV]) have been endorsed by a number of organizations including the Pharmacy Quality Alliance. ${ }^{10}$ Specialty pharmacies rely on such benchmarks to achieve accreditation, ${ }^{11,12}$ avoid reimbursement fees, and meet contracting requirements for payers, pharmacy benefits managers, and manufacturers, but current adherence calculations rely solely on pharmacy claims data. Sometimes patients discontinue or temporarily stop treatment for medically warranted reasons as advised by their health care team (i.e., misidentification of nonadherence); other times patients discontinue for reasons not directed by a health care provider (i.e., true nonadherence). Yet, methodology using claims data cannot distinguish these reasons for nonadherence because it does not recognize appropriate reasons for disruptions in therapy, creating the potential to inflate rates of nonadherence.

The negative effect of nonadherence on patient outcomes, pharmacies, and the health system calls for a better understanding of reasons for gaps in therapy, as well as calculations that better capture true nonadherence. Varying and complicated reasons for nonadherence to specialty medications must be further understood before effective interventions can be implemented. ${ }^{13,14}$ The purpose of this study was to identify rates and reasons for misidentified and true nonadherence in a diverse specialty patient population.

\section{Methods}

\section{Setting, Patient Population, and Data Source}

We performed a single-center, retrospective cohort study of patients filling 3 or more specialty prescriptions from Vanderbilt Specialty Pharmacy from March 1, 2017, to February 28,2018 . Vanderbilt Specialty Pharmacy, a health-system integrated specialty pharmacy, fills more than 5,000 prescriptions per month. Clinical pharmacists are embedded in specialty clinics with other members of the health care team, supporting patients and providers by ensuring appropriate use of and access to specialty medications. Data from the pharmacy dispensing system, EnterpriseRX, was collected and included adjudication date, days supply, product, and clinic assignment. This study received approval from the Vanderbilt University Institutional Review Board.

\section{Proportion of Days Covered Methodology}

Medication adherence was measured using the proportion of days covered (PDC) calculation along with Structured Query Language, which evaluated patient gaps in therapy versus overlapping days of medication coverage during the study period. Analytic functions were used to find the first fill date and last fill date of the medication for each unique patient and specialty product, allowing for the calculation of time between fills and the carry over supply at the beginning and end of each instance.

Gaps in patient therapy were determined after comparing the time between fills and the next fill date after any carryover present on the preceding fill. A gap in therapy could only be filled by a previous overlap. Overlapping days or excess days in therapy were not allowed to backfill previous gap days in order to accurately capture the amount of days a patient was not covered during the study time period.

Next, the remaining gap days were subtracted from the patient's time between fills and then compiled as the total days covered for that patient's therapy. Enrollment days were then calculated as the days remaining when the first fill date is subtracted from the last day of the reporting period. Final PDC was then determined at the patient and medication level by the total days covered divided by the enrollment period and multiplied by 100 to format value as a percentage.

\section{Adherence Calculation}

Patients were classified as nonadherent if their PDCs were less than $80 \%$. This threshold was selected because the Pharmacy Quality Alliance has suggested that the benchmark for adherence, a PDC of $80 \%$, is a threshold above which medications for many chronic disease states, including rheumatoid arthritis and multiple sclerosis, have a reasonable likelihood of achieving clinical benefit. Previous researchers have also used $80 \%$ as the threshold for adherence. ${ }^{15,16}$ Rates of nonadherence by clinic were calculated by dividing the number of nonadherent patients by the total included population. The RANDOM function in Excel (Microsoft, Redmond, WA) was used to select a $10 \%$ sample of nonadherent patients.

\section{Reason Categories}

Categories for nonadherence were initially drafted by the authors based on clinical experience. Potential categories were then discussed and updated based on feedback from a focus group of 10 specialty pharmacists and specialty pharmacy leadership. A community engagement studio was also held with several patients who had been treated with specialty medications. The studio was moderated and recorded by researchers with the goal of soliciting feedback from patients regarding their experiences with specialty pharmacies and exploring their ideas on potential reasons for nonadherence.

After compiling results from the focus group and community engagement studio, reasons for nonadherence were first categorized as "misidentified" or "true" and then further delineated within each category. Misidentified reasons for nonadherence categories and subcategories were defined as clinical (lab abnormalities, surgery/procedure, temporary contraindication, 
intolerance/adverse effect, other) or external fill (filled at another pharmacy, patient given samples, other). Categories and subcategories for true nonadherence reasons included financial (insurance change, affordability-loss of grant, affordability-high copay, other); clinical (intolerance/adverse effect, other); health literacy (medication safety/efficacy belief, alternative administration); memory (forgetfulness); unreachable (unreachable, declined contact); and patient unresponsive (unresponsive to getting labs, no show to appointments, unresponsive to financial office, failed to pick-up).

\section{Reasons for Nonadherence}

A clinical pharmacist and pharmacy intern conducted a chart review on a random $10 \%$ sample of nonadherent patients, searching multiple pharmacy and medical systems (i.e., pharmacy dispensing system, specialty pharmacy patient management system, and health system electronic medical record) for reasons for nonadherence. If a discrepancy in the reason for nonadherence arose, it was discussed among 3 researchers. All reasons for nonadherence found on chart review fit within the prespecified categories defined by the focus and community groups. If more than 1 potential reason for nonadherence was found for a specific patient, all identified reasons were counted.

\section{Results}

We included 7,488 prescriptions from 18 outpatient specialty clinics, representing 6,166 patients. The average PDC among the sample was $91.8 \%$. Of these patients, 1,059 (14.1\%) met criteria for nonadherence (PDC $<80 \%$ ). Among nonadherent patients, the average (standard deviation [SD]) age was 45 (20) years, and most were female (71.6\%) and Caucasian/white (88.6\%). The average PDC among nonadherent patients was $63.8 \%$ and ranged from $28.2 \%$ to $79.8 \%$. Specialty clinics with the highest average PDC included HIV (97.9\%), hepatitis C (97.2\%), and pulmonary arterial hypertension (96.7\%).

The randomly selected 10\% sample of nonadherent records represented 105 unique patients from 14 specialty clinics. The top 5 clinics represented in the sample size were rheumatology (29.7\%), oncology (17.8\%), multiple sclerosis (10.8\%), pediatric endocrinology (8.9\%), and gastroenterology and inflammatory bowel disease (7.2\%).

Table 1 outlines misidentified and true reasons for nonadherence identified from the chart review. Among the sample of nonadherent patients, $58 \%(n=61)$ were identified as truly nonadherent; $40 \%(n=42)$ were identified as misidentified as nonadherent; and 2\% $(n=2)$ were unknown. Multiple reasons for nonadherence were found in 21 patients.

The most common reason for true nonadherence was an inability of pharmacy staff to reach patients for refill calls ( $n=36,59 \%$ ), despite following the pharmacy's standard protocol for refill outreach (at least 3 phone calls followed by a letter to the patient). Other reasons for true nonadherence were due

\section{TABLE 1 Nonadherence Categorization of the} Sample $(N=105)$

\begin{tabular}{|c|c|c|}
\hline Nonadherence Category & & $(\%)$ \\
\hline \multicolumn{3}{|c|}{ True $(n=61)$} \\
\hline Unreachable & 36 & $(59.0)$ \\
\hline Unsuccessful phone attempt & 34 & $(55.7)$ \\
\hline Declined contact & 2 & $(3.3)$ \\
\hline Patient unresponsive & 10 & $(16.4)$ \\
\hline Labs not completed & & $(11.5)$ \\
\hline No show to appointment & 1 & $(1.6)$ \\
\hline Unresponsive to financial office & 1 & $(1.6)$ \\
\hline Failed to pick up & 1 & $(1.6)$ \\
\hline Health literacy & 10 & $(16.4)$ \\
\hline Medication safety/efficacy belief & 3 & $(4.9)$ \\
\hline Alternative administration & 7 & $(11.5)$ \\
\hline Financial & 10 & (16.4) \\
\hline Insurance change & 6 & $(9.8)$ \\
\hline Affordability: Loss of grant & 1 & $(1.6)$ \\
\hline Affordability: High copay & 2 & $(3.3)$ \\
\hline Other & 1 & $(1.6)$ \\
\hline Clinical & & $(13.1)$ \\
\hline $\begin{array}{l}\text { Intolerance/adverse effect } \\
\end{array}$ & & $(11.5)$ \\
\hline Other & 1 & $(1.6)$ \\
\hline Memory & 3 & $(4.9)$ \\
\hline Forgetfulness & 3 & $(4.9)$ \\
\hline \multicolumn{3}{|c|}{ Misidentified $(n=42)$} \\
\hline Clinical & 29 & $(69.0)$ \\
\hline Lab abnormalities & & $(9.5)$ \\
\hline Surgery/procedure & & $(16.7)$ \\
\hline Temporary contraindication & & $(14.3)$ \\
\hline Intolerance/adverse effect & 7 & $(16.7)$ \\
\hline Other & 5 & $(11.9)$ \\
\hline External fill & 13 & $(30.9)$ \\
\hline Filled at another pharmacy & & $(19.0)$ \\
\hline Patient given samples & 4 & (9.5) \\
\hline Other & 1 & $(2.4)$ \\
\hline \multicolumn{3}{|c|}{ Unknown $(\mathrm{n}=2)$} \\
\hline Unknown & & 2 \\
\hline $\begin{array}{l}\text { Note: Twenty-one patients had multi } \\
\text { number of reasons for nonadherence } \\
\text { represented in each category. }\end{array}$ & & $\begin{array}{l}\text { refore, } \\
\text { ents }\end{array}$ \\
\hline
\end{tabular}

to patient unresponsiveness ( $\mathrm{n}=10,16.4 \%$ ), low health literacy $(n=10,16.4 \%)$, and financial barriers $(n=10,16.4 \%)$. Of the 42 patients who were misidentified as nonadherent, 29 (69\%) were in response to clinical reasons or physician-driven decisions, and 13 (30.9\%) were dispensed by external pharmacies. Figure 1 depicts the reasons for misidentified and true nonadherence for each specialty clinic.

\section{Discussion}

\section{Adherence in a Specialty Population}

Adherence and appropriate use of specialty medications is an area of growing study and focus, since these agents continue 


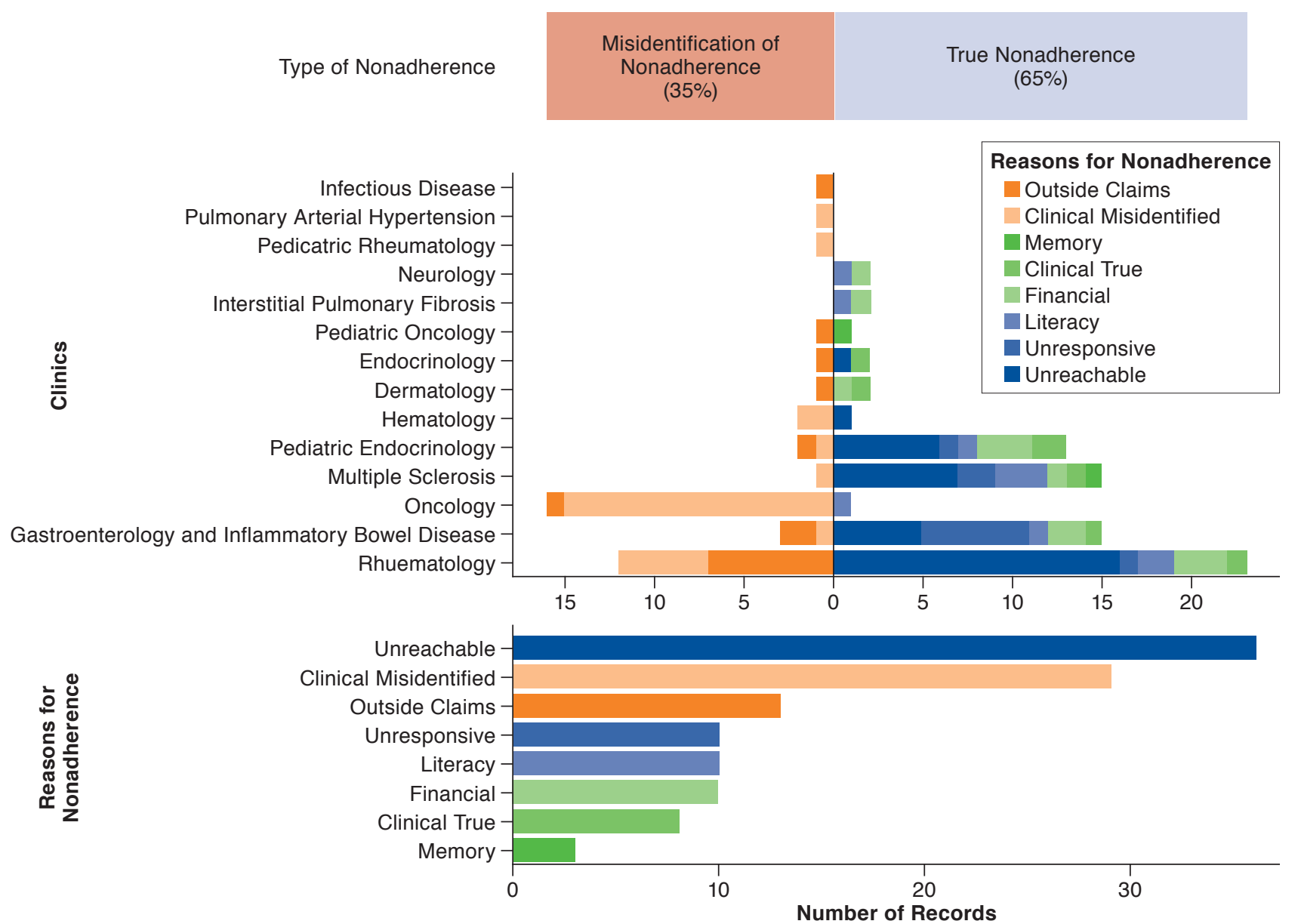

Note: This figure depicts the reasons for nonadherence by clinic and classification (e.g., misidentified vs. true). The first part conveys the ratio of misidentified nonadherence to true nonadherence. This also acts as a header for the mirrored histogram, such that everything to the left of the vertical line is misidentified nonadherence (warm colors) and to the right is true nonadherence (cool colors). The mirrored histogram depicts the reasons for nonadherence by clinic. The third part is a legend for the mirrored histogram, as well as a histogram of the reasons of nonadherence. Misidentified nonadherence accounts for 35\% of the recorded reasons for nonadherence (the 2 unknown reasons are not depicted in the figure). Clinical reasons (orange bars) were cited in 29 cases, 15 of which were within the Oncology Clinic. Within the Oncology Clinic, nonadherence was primarily classified as being misidentified, with only 1 patient having been recorded as being truly nonadherent (light blue = literacy). The

Rheumatology Clinic has the highest proportion of patients. Unreachable (dark blue) was the most frequently recorded reason for nonadherence within this clinic. Across all clinics, unreachable was the most common reason for nonadherence, with 36 instances. Memory was only cited as a reason for 3 instances of nonadherence.

to drive pharmacy costs. Specialty medications are more complicated than nonspecialty chronic treatments in their administration techniques, access barriers, and frequency of adverse effects and dosing adjustments. Better understanding of how these specialty-specific differences affect adherence is the first step to monitoring and improving use.

Across 18 specialty clinics, we found high overall rates of adherence, with $85.9 \%$ of patients achieving PDC $\geq 80 \%$. These findings affirm previous reports of high medication adherence in patients with specific specialty diseases enrolled in health system specialty pharmacies. ${ }^{17-19}$ Improved adherence in this model of care can be explained by improved access to specialty medications and high level of patient and provider engagement delivered through the integrated pharmacy services model. However, nonadherence persists in a high touch, integrated specialty pharmacy, with $14.1 \%$ of patients in our study not achieving the study definition of adherence.

Adherence rates varied among specialty clinic type. One potential explanation for these differences could be that the importance of a strict administration schedule correlating with prescribed days supply and prescription quantity is more important for some diseases than others. For instance, evidence suggests that a higher adherence threshold of $86 \%$ for adalimumab in treatment for inflammatory bowel disease is 
optimal for management of the disease. ${ }^{20}$ Therefore, measuring and targeting adherence might be effective by tailoring adherence thresholds to specific disease states and specialty medications. For example, a patient may be directed to hold an adalimumab dose when ill, or a patient may decide to skip a dose of a growth hormone due to discomfort with injections; each of these result in a gap in therapy. ${ }^{21}$ Using this information, a more personalized approach to calculating adherence could create a more accurate assessment of adherence behavior.

\section{Reasons for Nonadherence}

Understanding gaps in pharmacy claims provided additional insight into why patients appear nonadherent. For this study, we characterized misidentification of nonadherence as health care team-directed discontinuation or disruption in therapy. Outside pharmacy fills and clinical reasons for holding treatment were the main reasons for misidentification of nonadherence. Detailed evaluation of reasons for nonadherence revealed more than a third of patients who appeared nonadherent based on pharmacy claims calculations had clinically appropriate reasons for not following a prescribed administration schedule. This scenario was common in oncology patients, where doses may be held for a variety of reasons, including elevated lab markers, adverse reactions, and major decline in health status (Figure 1). Therefore, it is evident that calculating PDC based on pharmacy claims alone without accounting for misidentification of nonadherence may underestimate true adherence behavior.

We found that $12 \%$ of patients reviewed among patients with PDC less than $80 \%$ were due to the health system pharmacy no longer filling the prescription (i.e., outside claims). Patients are often required to transfer pharmacies due to payer or manufacturer restrictions, which unfortunately limits the ability of specialty pharmacies to adequately track adherence behavior and intervene when appropriate, since fill information for the external pharmacy is unavailable. Such restrictions introduce risk for gaps in therapy during pharmacy transitions and contribute to a false perception of nonadherence in specialty pharmacy reporting.

The relatively high rate of misidentified nonadherence and appropriate reasons for a large gap in fill data highlight the shortcomings of relying on pharmacy claims as a measure of adherence. Within this study's population, true adherence rates were underestimated due to a large portion of misidentified nonadherence. Although objective measures such as PDC are considered superior in accuracy to patient reports of adherence, ${ }^{13}$ appropriate reasons for nonadherence should be considered when creating benchmarks and contracting mandates that use pharmacy claims calculations as sole adherence metrics.

True nonadherence within our population was often due to pharmacy staff's unsuccessful phone attempts to reach a patient, with 34\% unreachable. Specialty pharmacies often rely on reaching patients by telephone for refills. However, specialty pharmacies need to focus on innovative, patientcentered communication modalities to better reach patients. Proposed methods such as text reminders have been associated with an increased adherence rate by as much as $16 \% \cdot{ }^{14,22,23}$ The use of a patient portal for refills, ${ }^{24}$ an electronic pillbox, ${ }^{25}$ and smartphone medication reminder applications ${ }^{26}$ have shown the ability to increase medication adherence. Creative patient contact methods, however, must be aligned with HIPAA privacy concerns.

Additional reasons for true nonadherence included patients being unresponsive to follow-up appointments or needed information such as labs or financial paperwork and having adverse effects. While closely following patients with scheduled phone calls and clinic visits, pharmacists can help address and manage adverse effects, if they are reported, to optimize treatment and improve disease control.

\section{Challenges of Measuring Adherence Using Pharmacy Claims}

The Pharmacy Quality Alliance recommends PDC as the preferred method to measure adherence. ${ }^{10}$ While PDC avoids an overestimation in adherence that occurs in other claims-based calculations, it does not adjust for appropriate gaps in therapy. ${ }^{13}$ PDC adherence calculations rely solely on pharmacy claims data, which do not account for health care team-directed discontinuation, disruption of treatment, or medication samples provided to the patient. Additionally, when PDC is calculated at the pharmacy level, fill data following a prescription transfer to an external pharmacy are unavailable to be included in the calculation. An inaccurate estimation of medication adherence can lead to several issues that are potentially costly to specialty pharmacies and unsafe to patients, including unnecessary labs, therapy adjustments, direct and indirect remuneration fees, and regulation charges. Our results indicate that a buffer to account for misidentified reasons for gaps in therapy when reporting PDC outcomes may be reasonable.

A high rate of true nonadherence was due to an inability to reach patients, low health literacy, and patients being unresponsive to follow-up appointments and requested information. This highlights the importance of thorough patient counseling at the time of treatment initiation. Multiple contact methods should be obtained, and patients should have a clear understanding of what will be needed to continue to access treatment. Further research on the best methods to perform such counseling and address specialty disease-specific reasons for nonadherence may provide additional insight on a more personalized approach to addressing nonadherence.

\section{Limitations}

This study has important limitations to consider. Our sample size for detailed review of reasons for nonadherence consisted of 105 patients, which is a small representation across the 
specialty clinics; therefore, additional reasons for nonadherence may not have been captured. Multiple platforms were used to identify reasons for nonadherence; however, all were reliant on prospective documentation of the reason for nonadherence, limiting the comprehensive collection of nonadherence reasons. In addition, this study was a single center, retrospective review of data limited to 1 year, from March 2017 through February 2018. We recognize that our demographic characteristics were limited to mostly white/Caucasian (88.6\%) and female $(67.6 \%)$ patients, likely driven by a high inclusion of patients with inflammatory conditions that meet this profile.

\section{Conclusions}

For patients identified as nonadherent based on pharmacy claims calculations, many are misidentified as nonadherent because of medically appropriate reasons. These findings highlight the need for improved adherence measures to more accurately identify incidence of medication nonadherence. In addition, efforts to improve adherence should focus on innovative methods to engage patients and provide effective counseling on the importance of ongoing communication with the pharmacy throughout treatment.

\section{Authors}

DOMINIC PAOLELLA, PharmD, Belmont University College of Pharmacy, Nashville, Tennessee. ELIZABETH CHERRY, PharmD; JACOB A. JOLLY, PharmD, CSP; JOSHUA DECLERCQ, MS; LEENA CHOI, PhD; and AUTUMN ZUCKERMAN, PharmD, BCPS, CSP, Vanderbilt Specialty Pharmacy, Vanderbilt University Medical Center, Nashville, Tennessee.

AUTHOR CORRESPONDENCE: Dominic Paolella, PharmD, Belmont University College of Pharmacy, 1900 Belmont Blvd., Nashville, TN 37212. Tel.: 815.793.6290;

E-mail:dominic.paolella@outlook.com.

\section{DISCLOSURES}

This study received funding support from CTSA Award No. UL1 TR002243 from the National Center for Advancing Translational Sciences. Study findings and conclusions are solely the responsibility of the authors and do not necessarily represent official views of the National Center for Advancing Translational Sciences or the National Institutes of Health. Zuckerman reports research support from Sanofi and Gilead Sciences, unrelated to this study. The other authors have nothing to disclose.

A poster based on the data from this study was presented at AMCP Nexus 2018 on October 24, 2018, in Orlando, FL.

\section{ACKNOWLEDGMENTS}

The authors acknowledge Jacob Bell for his contributions to identifying and extracting patient data; Megan Peter for her review of the abstract and manuscript; and the pharmacists of Vanderbilt Specialty Pharmacy who contribute to patient clinical care that makes this work possible. The authors also acknowledge the Vanderbilt Clinical and Translational Research (VCTR) Department for coordinating the patient engagement studio.

\section{REFERENCES}

1. Brown MT, Bussell JK. Medication adherence: WHO cares? Mayo Clin Proc. 2011:86(4):304-14.

2. Iuga AO, McGuire MJ. Adherence and health care costs. Risk Manag Healthc Policy. 2014;7:35-44.

3. De Geest S, Sabaté E. Adherence to long-term therapies: evidence for action. Eur J Cardiovasc Nurs. 2003;2(4):323.

4. Hirsch BR, Balu S, Schulman KA. The impact of specialty pharmaceuticals as drivers of health care costs. Health Aff (Millwood). 2014;33(10):1714-20.

5. Kale HP, Patel AM, Carroll NV. A comparison of pharmacy dispensing channel use and adherence to specialty drugs among Medicare Part D beneficiaries. J Manag Care Spec Pharm. 2018;24(4):317-26. Available at: https:// www.jmcp.org/doi/10.18553/jmcp.2018.24.4.317.

6. Waxman A, Chen SY, Boulanger L, Watson JA, Golden G. Factors associated with adherence to phosphodiesterase type 5 inhibitors for the treatment of pulmonary arterial hypertension. J Med Econ. 2013;16(2):298-306.

7. Munsell M, Frean M, Menzin J, Phillips AL. An evaluation of adherence in patients with multiple sclerosis newly initiating treatment with a self-injectable or an oral disease-modifying drug. Patient Prefer Adherence. 2017;11:55-62

8. Banks AM, Peter ME, Holder GM, et al. Adherence to disease-modifying therapies at a multiple sclerosis clinic: the role of the specialty pharmacist. J Pharm Pract. 2019:897190018824821.

9. Jacobs JM, Pensak NA, Sporn NJ, et al. Treatment satisfaction and adherence to oral chemotherapy in patients with cancer. J Oncol Pract. 2016;13(5):e474-e485.

10. Pharmacy Quality Alliance. PQA adherence measures. Updated August 28, 2018. Available at: https://www.pqaalliance.org/adherence-measures. Accessed September 28, 2019.

11. Accreditation Commission for Health Care. About accreditation. Available at: https://www.achc.org/about-accreditation.html. Accessed September 28, 2019.

12. Utilization Review Accreditation Commission. Specialty pharmacy accreditation. Available at: https://www.urac.org/programs/specialty-pharmacy-accreditation. Accessed September 28, 2019.

13. Lam WY, Fresco P. Medication adherence measures: an overview. Biomed Res Int. 2015;2015:217047.

14. Pop-Eleches C, Thirumurthy H, Habyarimana JP, et al. Mobile phone technologies improve adherence to antiretroviral treatment in a resourcelimited setting: a randomized controlled trial of text message reminders. AIDS. 2011;25(6):825-34

15. Raebel MA, Schmittdiel J, Karter AJ, Konieczny JL, Steiner JF. Standardizing terminology and definitions of medication adherence and persistence in research employing electronic databases. Med Care. 2013;51 (8 Suppl 3):S11-21.

16. Sodihardjo-Yuen F, van Dijk L, Wensing M, De Smet PA, Teichert M. Use of pharmacy dispensing data to measure adherence and identify nonadherence with oral hypoglycemic agents. Eur J Clin Pharmacol. 2016;73(2):205-13.

17. Bagwell A, Kelley T, Carver A, Lee JB, Newman B. Advancing patient care through specialty pharmacy services in an academic health system. J Manag Care Spec Pharm. 2017;23(8):815-20. Available at: https://www.jmcp. org/doi/10.18553/jmcp.2017.23.8.815.

18. Hanson RL, Habibi M, Khamo N, Abdou S, Stubbings J. Integrated clinical and specialty pharmacy practice model for management of patients with multiple sclerosis. Am J Health Syst Pharm. 2014;71(6):463-69.

19. Tschida S, Aslam S, Khan TT, Sahli B, Shrank WH, Lal LS. Managing specialty medication services through a specialty pharmacy program: the case of oral renal transplant immunosuppressant medications. J Manag Care Pharm. 2013;19(1):26-41. Available at: https://www.jmcp.org/doi/10.18553/ jmcp.2013.19.1.26. 
20. Govani SM, Noureldin M, Higgins PDR, et al. Defining an optimal adherence threshold for patients taking subcutaneous anti-TNFs for inflammatory bowel diseases. Am J Gastroenterol. 2018;113(2):276-82.

21. Rosenfeld RG, Bakker B. Compliance and persistence in pediatric and adult patients receiving growth hormone therapy. Endocr Pract. 2008;14(2):143-54.

22. Osterberg L, Blaschke T. Adherence to medication. N Engl J Med. 2005;353(5):487-97.

23. Thakkar J, Kurup R, Laba TL, et al. Mobile telephone text messaging for medication adherence in chronic disease: a meta-analysis. JAMA Intern Med. 2016;176(3):340-49.
24. Sarkar U, Lyles CR, Parker MM, et al. Use of the refill function through an online patient portal is associated with improved adherence to statins in an integrated health system. Med Care. 2014;52(3):194-201.

25. Welch G, Balder A, Zagarins S. Telehealth program for type 2 diabetes: usability, satisfaction, and clinical usefulness in an urban community health center. Telemed J E Health. 2015;21(5):395-403.

26. Santo K, Singleton A, Rogers K, et al. Medication reminder applications to improve adherence in coronary heart disease: a randomised clinical trial. Heart. 2019;105(4):323-29. 